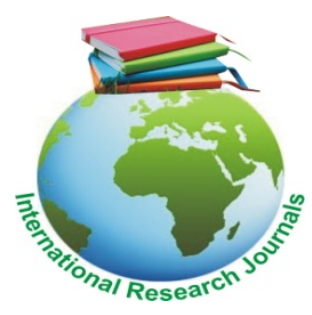

Journal of Medicine and Medical Sciences Vol. 7(3) pp. 047-055, May 2016

Available online http://www.interesjournals.org/JMMS

DOI: http:/dx.doi.org/10.14303/jmms.2016.302

Copyright (C) 2016 International Research Journals

\title{
Diabetes mellitus, associated co-morbidities and complications - A review
}

\author{
${ }^{1 *}$ Young E. E, ${ }^{2}$ Okafor C.N, ${ }^{3}$ Okwara C.C \\ ${ }^{1}$ Department of Medicine, University of Nigeria, Enugu Campus, Nigeria \\ ${ }^{2}$ Department of Physiology, University of Nigeria, Enugu Campus, Nigeria \\ ${ }^{3}$ Department of Medicine, University of Nigeria, Enugu Campus, Nigeria \\ *Corresponding author's E-mail: ekenechukwu.young@unn.edu.ng
}

\begin{abstract}
Diabetes mellitus is a metabolic disorder that results from a relative or absolute deficiency of insulin, which affects carbohydrate, protein and fat metabolism. Patients with diabetes mellitus may also have other comorbidities and complications, which require treatment. This review paper investigates the major comorbid conditions and complications that occur in patients with diabetes, their management and their influence on diabetes therapy. Evidence was gathered by a Medline search of original articles, review papers and theses on the prevalence and management of comorbidities and complications of diabetes as reported in the literature. Keywords included in the search were diabetes mellitus, comorbidities, and complications. Comorbidities and complications occur in every organ system in the body. Diabetic complications include neuropathy, nephropathy, retinopathy and cardiovascular disease. Their treatment includes the use of multiple medications. This results in poly-pharmacy, with some patients being prescribed as many as sixteen medications. The rational understanding of these comorbidities and complications and their management using recommended guidelines wouldresult in better outcomes.
\end{abstract}

Keywords: Diabetes mellitus, Comorbidities, Complications

\section{INTRODUCTION}

Diabetes mellitus is a disorder of metabolism in which an absolute or relative deficiency of insulin results in hyperglycaemia, leading to acute and chronic complications. The effects of diabetes mellitus involve complications in many organ systems in the body. In addition, in patients with type2 diabetes mellitus, especially the elderly, various co-morbidities may also coexist in them leading to high pill burden, increased morbidity and mortality, poor outcomes and low quality of life.

The Medical Expenditure Panel Survey reported that most adults with diabetes mellitus have at least one comorbidity (Druss et al., 2001) while up to $40 \%$ have at least three (Madiggan et al., 2005). Co-morbidities in diabetes have been classified into three groups by one author (Piette et al., 2006). These are:

1. Clinically dominant conditions; these are defined as comorbid conditions that are so serious that they eclipse the management of others e.g breast cancer, end-stage renal disease, class IV heart failure, severe depression etc.

2. Concordant versus discordant chronic conditions; Concordant conditions are considered as part of the same disease risk profile and are often part of the management profile e.g. hypertension, dyslipidaemia, coronary artery disease etc. Discordant conditions are not directly related in either pathophysiology or management e.g. asthma, arthritis etc.

3. Symptomatic versus asymptomatic chronic conditions; conditions such as depression, arthritis, gastrointestinal reflux disease are symptomatic, while hypertension, hyperlipidaemia are asymptomatic. In patients with Type1 diabetes, comorbidities may include the presence of other autoimmune diseases such as vitiligo, Addison's disease, rheumatoid arthritis etc. The chronic complications of diabetes mellitus occur as a result of several mechanisms, which result from the formation of advanced glycation end products, oxidative stress and 
endothelial damage. They are broadly classified into; a) Microvascular complications; retinopathy, neuropathy, nephropathy, foot disease.

b) Macrovascular complications; peripheral vascular disease, coronary artery disease, stroke, heart failure etc. Patients with diabetes also have increased risk of cancers such as breast cancer, pancreatic cancer and colorectal cancer. They also have increased risk of other disorders such as depression, cognitive decline, pancreatitis etc.

Hence in a patient with diabetes, it is important to consider the treatment of other comorbid conditions and complications when present, as they are likely to result in poor quality of life and adverse outcomes. However, this will require proper evaluation of treatment strategies, involvement of other specialties in management, consideration of pill burden, drug compliance and the possibility of multiplicity of adverse drug reactions and drug-drug interactions.

\section{METHODOLOGY}

A search of the literature was conducted using MEDLINE. Keywords included in the search were diabetes mellitus, comorbidities and complications. Articles on the comorbidities and complications including prevalence, pathophysiology and clinical features were identified and summarized.

\section{Comorbidities in diabetes mellitus and their treatment}

The presence of multiple comorbidities in a patient with diabetes can lead to frustrations among health care professionals attending to them due to lack of time during office visits to completely evaluate and manage each symptom and complaint (Grembowski et al.,., 2005). Comorbidities such as depression, arthritis, visual disturbances etc can also interfere with self-care activities by the patient and pose challenges to lifestyle changes and drug compliance. The common comorbidities encountered by patients can be classified according to organ systems.

\section{Respiratory system disorders}

\section{a) Asthma and Chronic obstructive pulmonary disease (COPD)}

Asthma and COPD are obstructive airway disorders, which result in airflow limitation, which in asthma has a reversible component. Asthma is also characterised by airway inflammation. Symptoms are episodic; characterised by wheeze, chest tightness and shortness of breath. Asthma tends to be genetic and may be classified as childhood or adult-onset asthma. The major risk factor for COPD is smoking. Other causative agents include cystic fibrosis, alpha antitrypsin deficiency, exposure to dusts and chemicals.

Ehrlich et al., have reported an increased incidence for asthma, COPD, pulmonary fibrosis and pneumonia in patients with diabetes than in the general population (Ehrlich et al., 2010). Potential problems may arise during the management of asthma or COPD comorbidity in patients with diabetes. The use of corticosteroids either as inhalation or orally for the management of asthma or COPD may lead to worsening of glycaemic control. Also, beta-blockers, which may be prescribed in the management of hypertension in diabetic patients, may precipitate an acute asthmatic attack in patients with both diseases.

\section{b) Lung infections}

Pneumonia is also more common in patients with diabetes (Grembowski ibid). Diabetes is known to result in disturbances in humoral innate immunity and an increased susceptibility to infections (Geerlings et al., 1999). It has been reported that there is increased risk of pneumonia-related hospitalizations in patients with both type 1 and type2 diabetes mellitus (Kornum et al., 2008). The treatment of pneumonia in patients with diabetes mellitus will require antibiotics with high sensitivity and possibly bactericidal action due to depressed immune function. The dosage of antibiotics used must be adjusted for renal function in patients with concomitant nephropathy and reduced renal function.

\section{Musculoskeletal disorders in diabetes mellitus}

Articular and periarticular manifestations are a frequent and often under diagnosed manifestation in patients with diabetes mellitus. There is reportedly increased incidence of diabetes mellitus and in the life expectancy of patients with diabetes mellitus. Expectedly an increase in the prevalence and clinical importance of many musculoskeletal disorders is seen. In elderly patients with diabetes mellitus, complaints of joint pains, swelling and limitation of movement are commonly due to the presence of various forms of arthritis. The prevalence of musculoskeletal disorders is not the same for type 1 and type $2 \mathrm{DM}$; being more common in those with longstanding type 1 DM (Peterson et al., 2001).

Some of the musculoskeletal complications with proven increased frequency among DM patients include:

(1) Diabetic cheiroarthropathy, (also known as diabetic stiff hand syndrome or limited joint mobility syndrome),

(2) Diffuse idiopathic skeletal hyperostosis (DISH) is characterized by metaplastic calcification of spinal 
ligaments (diagnosed on lateral spine radiographs) along with osteophyte formation. However, disc spaces, apophyseal joints, and sacroiliac joints are unaffected.

(3) Diabetic muscle infarction; a rare condition.

(4) Diabetic osteoarthropathy (also known as Charcot or neuropathic arthropathy). It is a condition involving destructive, lytic joint changes.

(5) Adhesive capsulitis, or frozen shoulder, has been reported in $19 \%$ of diabetic patients. This term refers to a stiffened glenohumeral joint usually caused by a reversible contraction of the joint capsule (Griggs et al., 2000, Balci et al., 1999).

(6) Calcific periarthritis of the shoulder is also seen in diabetes, where it is roughly three times more common than in people without diabetes.

(7) Early Osteoarthritis.

(8) Dupuytren's contracture results from a thickening, shortening, and fibrosis of the palmar fascia. It is reported in reported in $16-42 \%$ of diabetic patients (Rosenbloom A ed. 1997, Noble et al., 1984).

(9) Carpal tunnel syndrome.

(10) Flexor tenosynovitis (or trigger finger).

Of these, only limited joint mobility and stiff-hand syndrome occur exclusively in patients with diabetes (Rosenbloom et al., 1996).

Other musculoskeletal disorders that may be seen in DM patients are: reflex sympathetic dystrophy, also known as "shoulder-hand syndrome, rheumatoid arthritis, gout and non-specific arthralgia.

\section{Osteoarthritis}

The prevalence of osteoarthritis increases with age; hence it is expected to be a common comorbidity in elderly patients with diabetes. Osteoarthritis (OA) has been found to be more common in patients with diabetes. Advanced glycated end products (AGEs) formed in diabetic patients bind to chondrocytes in joints leading to production of inflammatory mediators (Zafar et al., 2011). When the OA occurs in the spine it is called spondylosis, which may be cervical or lumbosacral. Nerve root entrapment can occur as they exit the narrowed exit foramina and give rise to radicular pain.

The management of osteoarthritis includes pharmacological therapy with Non-steroidal antiinflammatory drugs (NSAIDs), opioid analgesics and acetaminophen. Chondroitin sulphate and glucosamine are also used to promote cartilage healing. NSAIDs must be used with caution in patients with sub-optimal renal function.

There is also a high risk of gastrointestinal bleeding with NSAIDs, which may be increased in elderly patients. Acetaminophen remains the first choice analgesic for elderly DM patients (Alan et al., 2010).

\section{Rheumatoid arthritis}

Rheumatoid arthritis (RA) has been linked with the development of cardiovascular disease (Mariana JK 2010). Rheumatoid arthritis is associated with chronic inflammation, which is known to be related to atherosclerosis. However, studies have not shown a direct association between rheumatoid arthritis and diabetes mellitus (Liao KP 2009).

Management issues arise because corticosteroid use for treating RA may further impair glycemic control or make it more difficult to control with blood glucose lowering agents. RA, DM and corticosteroids use are known risk factors for accelerated atherosclerosis and thus makes the affected patient more liable to develop cardiovascular events. Thus the lowest effective dose of corticosteroids and for the shortest duration of use is recommended in the DM patients with RA.

\section{Soft tissue rheumatism}

Soft tissue rheumatism is commoner among DM patients. The increased amount of advanced glycated end products (AGEs) formed in diabetic patients bind to the tendon sheets, ligaments and other soft tissue elements and lead to various soft tissue rheumatisms seen in this population of patients. These musculoskeletal manifestations of DM often go unrecognized and overlooked in daily clinical practice. There are published evidences of poor glycemic control predicting musculoskeletal disorders. Amongst 17 non-diabetic patients with rheumatic hand symptoms, 5 were found to have impaired glucose tolerance and 3 had frank diabetes (Baumgarten et al., 2007). Poor glycaemic control has not been shown to be a predictor of articular and periarticular manifestations in diabetes mellitus (Monnier et al., 1999). They reported that using the mean HbA1c level from results obtained during the last three visits they found no association between blood glucose control and MSK manifestations.

Thomas et al., also reported this relationship (2007). These findings are in sharp contrast to the results obtained in a British cohort that demonstrated a strong association between MSK manifestations and poor blood glucose control (Ramchun et al., 1986). It is of note that most musculoskeletal disorders are treatable especially early in their course, with resultant improvements in quality of life. It is therefore pertinent that medical practitioners be aware of the possible musculoskeletal complications of diabetes and evaluates for them, intervene and provide the best care for affected DM patients. At every clinic attendance the physician should endeavour to inquire from the DM patients about their symptoms and search for signs of musculoskeletal 
complications. This is an invaluable part of overall diabetes care.

\section{Diabetes mellitus and cardiovascular disease}

Cardiovascular disease is one of the common complications of diabetes mellitus. In developed countries, it is the most common cause of death in diabetic patients (Joron et al., 2002). Cardiovascular disease in diabetes is linked to the development of atherosclerosis. Patients with diabetes are classified as being high risk for cardiovascular disease, and diabetes mellitus is regarded as a 'coronary disease equivalent' (NCEP ATP III). The following are commonly associated with diabetes mellitus;

\section{a.) Hypertension}

Hypertension is common in patients with diabetes mellitus, with prevalence reported to be as high as between $50 \%$ to $75 \%$ (Colona et al., 2013). In patients with Type1 diabetes, hypertension is usually associated with diabetic nephropathy. In type2 DM, however it is associated with atherosclerosis and insulin resistance. The treatment of hypertension in diabetes is important to reduce the risk of cardiovascular events. The blood pressure goal is $130 / 80 \mathrm{mmHg}$ in diabetic patients. It is recommended that an ACE inhibitor or angiotensin receptor blocker should be used in these patients. Betablockers may mask hypoglycaemia in some patients and should be used with caution in patients with hypoglycaemia unawareness.

\section{b.) Heart failure}

Diabetes mellitus increases the risk of heart failure independent of the presence of hypertension or coronary heart disease. In the Framingham study, it was found that the risk of heart failure was 2.4 times higher in men and five times higher in women with diabetes (Kennel et al., 1974). Drugs such as thiazolidinediones should be avoided in patients with co-morbid diabetes and heart failure. Metformin should also be used with caution because of the risk of lactic acidosis.

\section{c.) Diabetic Cardiomyopathy}

The term "diabetic cardiomyopathy" was initially introduced based upon post-mortem findings in four diabetic adults who had heart failure in the absence of coronary heart disease (Rublen et al., 1972). Diabetic cardiomyopathy has been defined as ventricular dysfunction that occurs in diabetic patients independent of a recognized cause e.g coronary heart disease, hypertension (Zaroch et al., 1989). The pathogenesis is not fully understood.

\section{d.) Coronary artery disease and myocardial infarction}

Coronary artery disease is due to spasm or blockage of a coronary artery by atheroma. This leads to myocardial ischemia and may ultimately lead to infarction with death of cardiac muscle. Infarcts lead to poor myocardial contractility, cardiac remodelling, altered automaticity and rhythmicity. This can lead to heart failure, arrhythmias or sudden death. The underlying cause is atherosclerosis, which, as previously mentioned is common in patients with diabetes. The concomitant presence of cardiac autonomic neuropathy in diabetic patients may lead to denervation of the heart and 'silent' episodes of cardiac ischemia. Types 1 and 2 diabetes mellitus are independent risk factors for coronary heart disease (Wilson PW 1998).

Patients with diabetes are also at higher risk of mortality following a myocardial infarction. Treatment of coronary heart disease is similar to that in patients without diabetes. Calcium channel blockers and nitrates may be used. Beta-blockers can also be used. There is a higher mortality from $\mathrm{Ml}$ in diabetic patients than nondiabetics. In patients without previous DM, high blood glucose levels during an episode of $\mathrm{Ml}$ are also associated with high mortality rates. The management of inpatient hyperglycaemia is thus crucial to improve outcome in these patients. This is usually done with insulin.

\section{e) Stroke}

There is an increased risk of ischemic stroke in diabetic patients due to increased atherosclerosis. Stroke mortality is also increased almost 3-fold in diabetic patients (Stamler et al., 1983). Primary prevention is important by ensuring glycemic control, in addition to management of other risk factors. Antiplatelet drugs may be used for primary prevention in patients with multiple risk factors, and are beneficial in all patients with previous stroke for secondary prevention. Treatment of hypertension is essential. The use of statins is necessary in the primary and secondary prevention of stroke in patients who are not at target lipid levels. Recent guidelines have classified patients with diabetes mellitus as high risk and have recommended lower LDL goals of $<100 \mathrm{mg} / \mathrm{dl}$ in them( NCEP ATP III ). It is difficult to achieve this with only lifestyle measures; hence many will require statins, often in high doses.

Management of stroke in diabetes is by usual measures as in patients without diabetes. Early thrombolysis; < 3hours from onset of symptoms is 
beneficial in patients with ischemic stroke. Other supportive measures include nursing care, prevention of aspiration, maintenance of adequate mean arterial pressure and cerebral perfusion pressure, reduction of raised intracranial pressure if present and mobilization.

\section{Diabetes mellitus and neurological disorders}

\section{a) Cognitive dysfunction and dementia}

It has been found that patients with type2 diabetes mellitus often do poorly on measures of learning and memory (Mc Crimmon et al., 2012). Chronic hyperglycaemia and micro vascular disease contribute to cognitive decline. The incidence of dementia in diabetic patients has been reported to be two-to three fold higher than in non-diabetic subjects (Leibson et al., 1997).

The Diabetes Control and Complications Trial/Epidemiology of Diabetes Interventions and Complications (DCCT/EDIC) study, a long-term study that found that a decline in cognitive function, such as motor speed and psychomotor efficiency, was associated with glycemic control level in patients with type 1 diabetes who were followed up for approximately 18 years (Jose et al., 2001).

A modest association has been reported between Alzheimer's disease and diabetes mellitus (Sasaki et al., 1998). It is believed that deposition of advanced glycated end products is contributory (Harris MI 1998). However, some studies have not shown any association. Diabetes also increases the risk of vascular dementia and strokeassociated dementia. This is due to the presence of traditional cardiovascular risk factors such as obesity, hypertension and dyslipidaemia (Mc Corry et al., 2006).

\section{b) Epilepsy}

An association between epilepsy and diabetes has been recorded. In a study by a UK group, adults with epilepsy had a four-fold higher prevalence of type $1 \mathrm{DM}$ than the general population (Schober et al.,). A significantly higher prevalence of epileptic seizures was also found in children and adolescents with diabetes in another study. There was also an increased risk of diabetic ketoacidosis in patients who had both epilepsy and type1 DM.

\section{Cancer in patients with diabetes mellitus}

Diabetic patients are said to have a higher risk of cancers of the urinary tract, pancreas, liver, biliary tract, colon, endometrium and kidney than the general population (Habib et al., 2013). Diabetes and cancer also have several risk factors in common including age, ethnicity, obesity, alcohol, smoking and physical inactivity.
Hyperglycemia has been shown to be associated with cancer in prospective studies (Habib et al., ibid). In a study conducted on 12,792 participants categorized in relation to HbA1c levels, with median 15-year follow-up, elevated HbA1c levels were associated with higher cancer risk (Joshu et al., 2012). Diabetes is associated with higher incidence of hepatocellular carcinoma (Wang et al., 2012). It is postulated that increase of IGF-1 can stimulate cellular proliferation, inhibit apoptosis and promote carcinogenesis (Weinecke et al., 2004). Nonalcoholic steatohepatitis (NASH) is common in patients with diabetes and is a significant risk factor for hepatocellular carcinoma. The relative risk imposed by DM is greatest (about 2-fold or higher) with regard to liver, pancreas, and endometrium cancer and lower for cancers of kidney, bladder, breast, colorectal, oesophagus, biliary tract, and lymphoma.

Recently, epidemiologic, in vitro and in vivo model studies have reported antineoplastic properties of metformin (Martin and Marais 2012). Metformin lowers cancer risk by $39 \%$ when compared to other therapies (Yan et al., 2012). The actions of metformin include mTOR inhibition, cyclin D1 and p53 interference. Metformin has also been shown to repress the proliferation and reduce survival of cancer stem cells (Rattan et al., 2012).

\section{Diabetes mellitus and gastrointestinal disorders}

Gastrointestinal disorders may be present in patients with diabetes, either as a co-morbidity or as a complication.

a.) Peptic ulcer disease; Peptic ulcer disease (PUD) and also non-ulcer dyspepsia are common disorders in the general population and as such may also be seen in patients with diabetes mellitus.

b.) Gastroesophageal reflux disease: GERD is also common in the general population and not specifically related to diabetes. Obesity is a risk factor for reflux and also for diabetes. Management involves the use of proton pump inhibitors or $\mathrm{H} 2$ receptor blockers. Lifestyle measures such as weight loss, sleeping propped up, reduction of alcohol intake, intake of smaller meals, reducing caffeine etc are also helpful.

c.) Fatty liver: The incidence of fatty liver i.e. nonalcoholic fatty liver disease is higher in patients with diabetes. It is regarded in some people as the hepatic complication of diabetes. Some studies have reported that the prevalence of NAFLD is up to $70 \%$ in patients with diabetes using ultrasonography and liver biopsy (Williams et al., 2011). Fatty liver can progress to nonalcoholic steatohepatitis (NASH), which is a risk factor for liver cirrhosis and hepatocellular carcinoma. Treatment is with metformin and a thiazolidinedione.

d.) Gastroparesis: this is a feature of diabetic autonomic neuropathy. It presents as bloating, easy satiety and nausea. Treatment is with erythromycin, metoclopramide 
or cisapride. Pacing of the stomach may be needed in severe cases.

\section{Diabetes mellitus and infections}

Infections are common in patients with diabetes mellitus and occur due to metabolic derangements. Hyperglycaemia results in impaired humoral immunity and leukocyte function (Delamaire et al., 1997). The most common sites of infection are the skin and the urinary tract. Lower urinary tract infections and pyelonephritis are more common in them. Malignant otitis externa, rhinocerebralmucormycosis, and emphysematous pyelonephritis, occur almost exclusively in patients with diabetes. Diabetes is a risk factor for the reactivation of tuberculosis. Systematic reviews show that TB risk is at least tripled in patients with diabetes, especially in younger patients. It is also associated with worse TB outcomes (Stevenson et al., 2007).

\section{Diabetes mellitus and mental disorders}

Mental health issues tend to be commonly overlooked in patients and only about one-third with mental comorbidities receives treatment (Ducat et al., 2014). The Diabetes Attitudes, Wishes and Needs (DAWN) study was the world's largest international study on psychosocial aspects of diabetes (Peyrot et al., 2005). The study showed that as many as $41 \%$ of the patients had poor psychological well being, however only $10 \%$ received any psychological care. Psychological distress in diabetic patients is associated with screening, introduction of lifestyle changes and drug treatment (Young EE et al., 2012). Diabetes is associated with a two-fold increased risk of depression (Anderson et al., 2001). Other mental health comorbidities include eating disorders and anxiety disorders (Ducat et al., ibid).

Major depressive disorder may occur in patients with diabetes. Youths with Type1 DM have been found to have higher rates of depression. 'Diabetes distress' has also been described as a separate entity. It occurs because of the high level of self-management required by patients in caring for their diabetes. This requires a complex set of behavioural tasks by the patient, family and friends every single day. Diabetes-related distress includes feeling overwhelmed, fear about the future and complications of the disease and feeling guilty when management goals are not met (Ducat et al., ibid).

\section{Chronic complications of diabetes mellitus and their management}

Diabetes mellitus is associated with chronic complications, which increase the risk of mortality and are associated with poor quality of life. The central pathogenic mechanism in the development of chronic DM complications is the presence of chronic hyperglycaemia, which acts through several mechanisms, these include;

i) Formation of advanced glycation end products (AGEs).

ii) Increased flux of glucose through the polyol pathway leading to production of sorbitol.

iii) Activation of protein kinase $C$.

iv) Increased hexosamine pathway.

These lead to damage of tissues via oxidative stress, vascular permeability, angiogenesis etc.

\section{Diabetic neuropathy}

Diabetic neuropathy is a descriptive term meaning a demonstrable disorder, either clinically evident or subclinical, which occurs in the setting of diabetes mellitus WITHOUT other causes of peripheral neuropathy. The neuropathy disorder includes manifestations in the somatic and/or autonomic nervous system (ADA 1998). It occurs in up to $50 \%$ of patients with diabetes. It is a major cause of increased morbidity and mortality and is associated with poor quality of life. Neuropathy can also occur in persons with impaired glucose tolerance and metabolic syndrome. Diabetic peripheral neuropathy increases the risk of amputation 1.7 fold and 12 fold if there is deformity. Diabetic neuropathy is a neurological complication that affects all parts of the nervous system; both autonomic and somatic nerves. Nerve hypoxia leads to structural damage and reduced nerve conduction velocity. Micro vascular defects in the endoneurial vessels, such as gross basement membrane thickening, endothelial cell proliferation and hypertrophy as well as reduced oxygen tension are observed.

Risk factors for diabetic peripheral neuropathy include age, height, poor glycaemic control, longer duration of diabetes, increased triglycerides, smoking, hypertension and obesity. The onset is usually insidious, however may be acute.

Positive symptoms include tingling, pain, prickling sensations. Negative symptoms include numbness, "asleep" etc. Symptoms occur in a glove and stocking pattern; starts in feet, tend to be symmetrical and may be associated with neuropathic oedema and wasting if severe.

Autonomic neuropathy presents with symptoms in any area of the autonomic system, including cardiac autonomic neuropathy, which is associated with arrhythmias, silent ischemia and sudden death. Patients with autonomic neuropathy present with resting tachycardia, postural hypotension, gastroparesis, urine incontinence, erectile dysfunction, nocturnal diarrhea etc. Table1 shows common drugs used to treat various manifestations of diabetic autonomic neuropathy.

The management involves supportive measures, counselling, ensuring strict glycaemic control, treatment 
Table1: Treatment of symptoms of autonomic neuropathy

\begin{tabular}{lc}
\hline SYMPTOM & TREATMENT \\
\hline Gastroparesis & Metoclopramide, Domperidone, \\
Diarrhoea & Erythromycin, Gastric pacemaker \\
Postural dizziness & Loperamide, antibiotics, clonidine, octreotide \\
Excessive sweating & Fludrocortisone, Support stockings, midodrine, NSAIDs \\
Erectile dysfunction & Anticholinergicse.gpropantheline, clonidine \\
& PDE5 inhibitors eg Sildenafil, Tadalafil. \\
& Penile prostheses \\
Vacuum devices \\
Penile prostheses
\end{tabular}

Table 2: Stages of diabetic nephropathy

\begin{tabular}{lcc}
\hline Stage & Urine dipstick & Urine albumin:creatinine ratio \\
\hline Normal & Negative & $<2$ \\
Microalbuminuria & Negative & $2-20$ \\
Overt & Positive & $>20$ \\
\hline
\end{tabular}

of hypertension and dyslipidaemia. Peripheral neuropathy is difficult to treat. Useful drugs include tricyclic antidepressants, anti-convulsants such as gabapentin, pregabalin, carbamazepine, anti-oxidants such as lipoic acid etc.

Patients with neuropathy are advised to care for their feet to avoid foot ulcers.

\section{Diabetic nephropathy}

Diabetic nephropathy is one of the dreaded complications of diabetes. It is one of the common causes of end-stage renal disease requiring dialysis or transplantation.

Diabetic nephropathy presents as increased urinary protein. In the early stages, there is microalbuminuria, which later progresses to overt proteinuria. The prevalence of diabetic nephropathy is higher in African Americans, Asians and nativeAmericans than in Caucasians (Young et al., 2003). Diabetic nephropathy is classified into five stages based on urinary albumin excretion (Table 2). Patients with microalbuminuria may either progress to macroalbuminuria or regress to normoalbuminuria. Screening is done by measuring the albumin: creatinine ratio in a spot urine sample. The glomerular filtration rate (GFR) should also be estimated. It is important to rule out non-diabetic kidney disease, which can occur in up to $12-38 \%$ of patients (Christensen et al., 2001). The risk factors for diabetic nephropathy include hypertension, dyslipidaemia, smoking and genetic factors (Zolmanovitz et al., 2009).
Histologically, the lesion is thickened glomerular basement membrane and glomerulosclerosis with the resultant 'Kimmelstiel-Wilson' lesion.

Treatment involves primary and secondary prevention. A multiple risk factor interventional approach is beneficial, including treatment of hypertension, dyslipidaemia and hyperglycaemia, smoking cessation etc. Proteinuria can be effectively reduced with ACE inhibitors or Angiotensin receptor blockers ARBs. Intensive treatment of hypertension to below $130 / 80 \mathrm{mmHg}$ or $125 / 75 \mathrm{mmHg}$ in those with proteinuria is beneficial. Metformin is contraindicated when serum creatinine is above $1.5 \mathrm{mg} / \mathrm{dl}$ in men and $1.4 \mathrm{mg} / \mathrm{dl}$ in women due to the increased risk of lactic acidosis (Bailey et al., 1996). Sulfonylureas are mainly excreted in the kidney and have to be used with caution. The presence of renal disease leads to a reduced clearance of insulin and prolonged effect, hence this should be noted. Other drugs should also be used with care in patients with renal disease.

\section{Diabetic retinopathy}

Diabetic retinopathy begins with micro-aneurysms, progressing to exudative changes, ischaemia, oedema, macular oedema and proliferative changes. Mild to moderate retinopathy leads to problems with visual acuity, visual fields, reading, driving etc.

Intensive diabetic control effectively reduces the risk of retinopathy in patients with diabetes. Renin-angiotensin system inhibitors reduce the incidence and risk of 
progression of diabetic retinopathy in persons with type1 diabetes (Mauer et al., 2009).

Fenofibrate also reduces the risk of progression by $40 \%$. Eye-specific treatments can be medical or surgical. The VEGF-neutralizing antibodies bevacizumab and ranibizumabhave been shown to improve visual acuity by an average of one to two lines on a Snellen chart. Other therapies include intravitrealfluocinolone and laser photocoagulation.

\section{Poly-pharmacy in diabetes mellitus}

The comprehensive management of patients with diabetes mellitus includes lowering of the blood glucose, management of associated cardiovascular risk factors, any comorbidities and complications.

This often results in the prescription of a large number of different molecules in diabetic patients and raises the issue of compliance in patients.

One study reported that people with type2 DM were prescribed $8.4 \pm 3.0$ different drug compounds per day (up to a maximum of 16 ) and took $8.6 \pm 3.9$ tablets per day (up to a maximum of 22) (Bauers 2014). However, over $97 \%$ of the medications were according to recommended guidelines.

Grant et al., found that patients with diabetes had high adherence rates, despite the large numbers of medications prescribed (Grant et al., 2003). A higher level of poly-pharmacy was reported to be associated with a higher medication adherence than the nonpolypharmacy cohort, despite higher economic costs (Chen et al., 2013). However, adherence declines in patients on multiple daily dose regimens compared to once daily regimens (Donnan et al., 2002).

\section{CONCLUSION}

Diabetes mellitus is a metabolic disorder, which is associated with several comorbidities and complications affecting every organ system. Proper management usually requires a multi-disciplinary approach to address this.

Poly-pharmacy is common and appropriate in many patients to achieve treatment goals, reduce or prevent complications and improve the quality of life of patients.

\section{REFERENCES}

American Diabetes Association and American Academy of Neurology. Consensus statement (1998). Report and recommendations of the San Antonio conference on diabetic neuropathy. Diabetes Care; 11: 592-597.

Alan DK, Amir B, Jared TS (2010). Pain Management in the Elderly Population: A Review. Ochsner J. Fall; 10(3): 179-187.

Anderson RJ, Freedland KE, Clouse RE (2001). The prevalence of comorbid depression in adults with diabetes. Diabetes Care; 24:
1069-1078.

Bailey CJ, Turner RC (1996). Metformin. N Engl J Med, 334:574-579.

Balci N, Balci MK, Tuzuner S (1999). Shoulder adhesive capsulitis and shoulder range of motion in type II diabetes mellitus: association with diabetic complications. J Diabetes Complications;13:135-40.

Bauer S, Nauck MA (2014). Polypharmacy in people with type1 and type2 diabetes is justified by current guidelines- a comprehensive assessment of drug prescriptions in patients needing inpatient treatment for diabetes-associated problems. Diabetic Med; 31(9): 1078-1085.

Baumgarten KM, Gerlach D, Boyer MI (2007). Corticosteroid injection in diabetic patients with trigger finger. A prospective, randomized, controlled double-blinded study. J Bone Joint Surg.; 89:2604-2611.

Chen SY, Alas V, Lee YC, Greene M, Oderda G (2013). Evaluation of polypharmacy in patients with type2 diabetes mellitus and its association with medication adherence and health care costs. Value in Health; 16(3): A173.

Christensen PK, Larsen S, Horn T, Olsen S, Parving HH (2001). Renal function and structure in albuminuric type 2 diabetic patients without retinopathy. Nephrol Dial Transplant, 16:2337-2347.

Colosia AD, Palencia R, Khan S (2013). Prevalence of hypertension and obesity in patients with type 2 diabetes mellitus in observational studies: a systematic literature review. Dovepress; 6: 327-338.

Delamaire $M$, Maugendre $D$, Moreno $M$, Le Goff MC, Allannic $H$, Genetet B (1997). Impaired leucocyte functions in diabetic patients. Diabet Med. Jan; 14(1):29-34.

Donnan PT, MacDonald TM, Morris AD (2002). Adherence to prescribed oral hypoglycemic medication in a population of patients with type2 diabetes; a retrospective cohort study. Diabetic Med; 19: 279-284.

Druss BG, Marcus SC, Olfson M, Tanielian T, Elinson L, Pincus HA (2001). Comparing the national economic burden of five chronic conditions. Health Aff (Millwood) 20: 233-241,

Ehrlich FS, Quesenberry CP, Van DenEeden SK, Ferrara A (2010). Patients Diagnosed With Diabetes Are at Increased Risk for Asthma, Chronic Obstructive Pulmonary Disease, Pulmonary Fibrosis, and Pneumonia but Not Lung Cancer. Diabetes Care Diabetes Care 33: 155-60.

Geerlings SE and Hoepelman AIM (1999). Immune dysfunction in patients with diabetes mellitus. FEMS Immunology and Med Microb 26: 259-265.

Grant RW, Denta NG, Singer DE, Meigs JB (2003). Polypharmacy and medication adherence in patients with type2 diabetes. Diabetes Care; 26(5): 1408-1412.

Grembowski D, Paschane D, Diehr P, Katon W, Martin D, Patrick DL (2005). Managed care, physician job satisfaction, and the quality of primary care. J Gen Intern Med 20: 271-277.

Griggs SM, Ahn A, Green A (2000). Idiopathic adhesive capsulitis. A prospective functional outcome study of nonoperative treatment. J Bone Joint Surg (Am);82:1398-407.

Habib SL, Rojna M (2013). Diabetes and risk of cancer. ISRN Oncology Vol. Article ID 583786, 16 pages http://dx.doi.org/10.1155/2013 1583786 .

Harris MI. Diabetes in America (1998). Epidemiology and scope of the problem. Diabetes Care 21(suppl 3):C11-14.

Jose A. Luchsinger, Ming-Xin Tang (2001). Yaakov Stern Diabetes Mellitus and Risk of Alzheimer's Disease and Dementia with Stroke in a Multiethnic Cohort. Am J Epidem; 154(7): 635-641.

Joshu CE, Prizment AE, Dluzniewski PJ (1986). "Glycatedhemoglobin and cancer incidence and mortality in the Atherosclerosis in Communities (ARIC) Study, 1990-2006," Intl. J. of Cancer X;131 (7):1667-1677.

Joron JE, Laryea E, Jaegen D, Macdonald L (1986). Causes of death in 1144 patients with diabetes mellitus: an autopsy study. CMAJ;134: 759-764.

Kannel WB (1974). Hjortland M, Castelli WP. Role of diabetes in congestive heart failure: the Framingham study.Am $J$ Cardiol.;34(1):29.

Kornum JB, Thomsen RW (1974). Riis A, Lervang HH, Schønheyder HC, SørensenHT: Diabetes, glycemic control, and risk of hospitalization with pneumonia: a population-based case-control study. Diabetes Care X; 31: 1541- 1545. 
Lee Ducat, Louis H. Philipson, Barbara J (2014). Anderson. The mental health comorbidities of diabetes. JAMA; 312(7): 691-692.

Leibson CL, Rocca WA, Hanson VA (1997). Risk of dementia among persons with diabetes mellitus: a population-based cohort study. Am J Epidemiol; 145: 301-308.

Liao KP (2009). A specific association exists between type 1 diabetes and anti-CCP positive rheumatoid arthritis. Arthritis Rheum.; 60:653660.

Maddigan SL, Feeny DH, Johnson JA (2005). Health-related quality of life deficits associated with diabetes and comorbidities in a Canadian National Population Health Survey. Qual Life Res 14: 1311-1320.

Mariana J Kaplan (2010). Cardiovascular complications of Rheumatoid Arthritis - Assessment, prevention and treatment. Rheum Dis Clin North Am.; 36: 405-426.

Martin M, Marais R (2012) "Metformin: a diabetes drug for cancer, or a cancer drug for diabetics?" Journal of Clinical Oncology; 309(21): 2698-2700.

Mauer M, Zinman B, Gardiner R (2009). Renal and retinal effects ofenalapril and losartan in type1 diabetes. N Engl J Med 361:40-51.

Mc Corry D, Nicolson A, Smith D (2006). An Association between Type 1 Diabetes and Idiopathic Generalized Epilepsy. Ann Neurol; 59: 204-6.

Mc Crimmon RJ, Ryan CM, Frier BM (2012). Diabetes and cognitive dysfunction. The Lancet 379(9833): 2291-2299.

Monnier VM, Bautista O, Kenny D, Sell DR, Fogarty J, Dahms W (1999). Skin collagen glycation, glycoxidation, and cross linking are lower in subjects with longterm intensive versus conventional therapy of type 1 diabetes. Diabetes;48:870-80.

National Cholesterol Education Program (NCEP) (2002). Expert Panel on Detection, Evaluation, and Treatment of High Blood Cholesterol in Adults (Adult Treatment Panel III): Third Report of the National Cholesterol Education Program (NCEP) Expert Panel on Detection, Evaluation, and Treatment of High Blood Cholesterol in Adults (Adult Treatment Panel III) final report. Circulation 106:3143-3421.

Noble J, Heathcote JG, Cohen H (1984). Diabetes mellitus in the aetiology of Dupuytren's disease. J Bone Joint Surg (Br);66:322-5).

Peterson R, Edelman SV, Kim DD (2001). Musculoskeletal Complications of Diabetes Mellitus. Clinical Diabetes.; 19:132-135.

Piette JD, Kerr EA (2006). The Impact of Comorbid Chronic Conditions on Diabetes Care. Diabetes Care; 29: 725-731.

Peyrot M, Rubin RR, Lauritzen T (2005). Psychosocial problems and barriers to improved diabetes management: results of the CrossNational Diabetes Attitudes, Wishes and Needs (DAWN) Study. Diabetic Med.; 22: 1379-1385.

Ramchurn N, Mashamba C, Leitch E, Arutchelvam V, Narayanan K, Weaver JN (2009). Upper limb musculoskeletal abnormalities and poor metabolic control in diabetes. Eur J Intern Med;20:718-21.

Rattan R. Fehmi A, Munkarah A (2012). "Metformin: an emerging new therapeutic option for targeting cancer stem cells and metastasis," Journal of Oncology, Article ID 928127, 12 pages.

Rosenbloom A. Connective tissue disorders in diabetes (1997). International textbook of diabetes mellitus. 2 nd ed. Chichester: John Wiley,:1517-31.

Rosenbloom AL, Silverstein JH (1996). Connective tissue and joint disease in diabetes mellitus.EndocrinolMetabClin North Am.; 25:473-83.

Rubler S, Dlugash J, Yuceoglu YZ (1972). New type of cardiomyopathy associated with diabetic glomerulosclerosis.Am J Cardiol. 30(6):595.

Sasaki N, Fukatsu R, Tsusuki K (1998). Advanced glycation end products in Alzheimer's disease and other neurodegenerative diseases. Am J Pathol; 153:1149-55.
Schober E, Otto KP, Dost: A for the German/Austrian DPV Initiative and the BMBF competence network diabetes. Association of epilepsy and type 1 diabetes in children and adolescents. Is there an increased risk for DKA? J. of Pediatrics (in press).

Stamler J, Vaccaro O, Neaton JD, Wentworth D (1993). Diabetes, other risk factors, and 12-year cardiovascular mortality for men screened in the Multiple Risk Factor Intervention Trial (MRFIT). Diabetes Care;16:434-444.

Stevenson CR, Critchley JA, Forouhi NG (2007). Diabetes and the risk of tuberculosis: a neglected threat to public health? Chronic IIIn; 3 : 228-45.

The Diabetic Retinopathy Clinical Research Network (2010). Randomized trial evaluating ranibizumab plus prompt or deferred laser or triamcinolone plus prompt laser for diabetic macular edema. Ophthalmology;117(6):1064.e35-1077.e35.

Themis Z, Fernando G, Amely PSB, Fúlvio CST, Jorge DM, Luís H (2009). Canani Diabetic Nephropathy. DiabMetabSynd; 1: 10.

Thomas SJ, McDougall C, Brown ID, Jaberoo MC, Stearns A, Ashraf R (2007). Prevalence of symptoms and signs of shoulder problems in people with diabetes mellitus. J Shoulder Elbow Surg;16:748-51.

Wang C, Wang X, Gong G (2012) "Increased risk of hepatocellular carcinoma in patients with diabetes mellitus: a systematic review and meta-analysis of cohort studies," International Journal of Cancer; 130 (7): 1639-1648.

Wiencke JK (2004). "Impact of race/ethnicity onmolecular pathways in human cancer," Nature Reviews Cancer 4(1) 79-84.

Williams C D, Stengel J, AsikeM I (2011). "Prevalence of nonalcoholic fatty liver disease and nonalcoholicsteatohepatitis among a largely middle-aged population utilizing ultrasound and liver biopsy: a prospective study," Gastroenterology 140(1): 124-131.

Wilson PW (1998). Diabetes mellitus and coronary heart disease. Am J Kidney Dis;32:S89 -S100.

Yan K H, Yao C J, Chang H Y (2010). "The synergistic anticancer effect of troglitazone combined with aspirin causes cell cycle arrest and apoptosis in human lung cancer cells," Molecular Carcinogenesis; 49(3): 235-246.

Young BA, Maynard C, Boyko EJ (2003). Racial differences in diabetic nephropathy, cardiovascular disease, and mortality in a national population of veterans.Diabetes Care, 26:2392-2399.

Young EE, Unachukwu CN (2012). Psychosocial aspects of diabetes mellitus. AJDM; 20(1): 5-7.

Zafar R, Nahid A, Tariq MH (2011). Advanced glycation end products induce the expression of interleukin- 6 and interleukin- 8 by receptor for advanced glycation end product- mediated activation of mitogenactivated protein kinases and nuclear factor- $k B$ in human osteoarthritis chondrocytes. Rheumatology.; 50: 838-851.

Zarich SW, Nesto RW (1989). Diabetic cardiomyopathy. Am Heart J.118(5 Pt 1):1000. 